\title{
Inverse Photonic Glasses by Packing Bidisperse Hollow Microspheres with Uniform Cores
}

\section{Citation}

Seung-Hyun Kim, Sofia Magkiriadou, Do Kyung Rhee, Doo Sung Lee, Pil J. Yoo, Vinothan N. Manoharan, Gi-Ra Yi. 2017. Inverse Photonic Glasses by Packing Bidisperse Hollow Microspheres with Uniform Cores. ACS Applied Materials \& Interfaces 9, no. 28: 24155-24160.

\section{Published Version}

10.1021/acsami.7b02098

\section{Permanent link}

http://nrs.harvard.edu/urn-3:HUL.InstRepos:42641435

\section{Terms of Use}

This article was downloaded from Harvard University's DASH repository, and is made available under the terms and conditions applicable to Open Access Policy Articles, as set forth at http:// nrs.harvard.edu/urn-3:HUL.InstRepos:dash.current.terms-of-use\#OAP

\section{Share Your Story}

The Harvard community has made this article openly available.

Please share how this access benefits you. Submit a story.

\section{Accessibility}


This document is the Accepted Manuscript version of a Published Work that appeared in final form in ACS Applied Materials \& Interfaces, copyright (C) American Chemical Society after peer review and technical editing by the publisher. To access the final edited and published work see http://pubs.acs.org/articlesonrequest/AOR-pCMFR6UTq9WREhISfTfT

\section{Inverse Photonic Glasses by Packing Bi-disperse Hollow Microspheres with Uniform Cores}

Seung-Hyun Kim, ${ }^{\dagger}$ Sofia Magkiriadou, ${ }^{\S}$ Do Kyung Rhee, ${ }^{\dagger}$ Doo-Sung Lee ${ }^{\dagger}$, Pil J. Yoo, ${ }^{\dagger, \dagger}$ Vinothan N. Manoharan, ${ }^{\#, \S}$ Gi-Ra Yi ${ }^{\dagger, *}$

†School of Chemical Engineering and ${ }^{\ddagger}$ Sungkyun Advanced Institute of Nano Technology (SAINT), Sungkyunkwan University, Suwon 16419 Republic of Korea

${ }^{\S}$ Department of Physics and ${ }^{\#} J o h n$ A. Paulson School of Engineering and Applied Sciences, Harvard University, Cambridge, USA

*E-mail: yigira@skku.edu

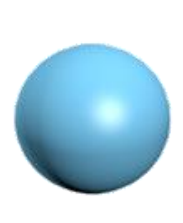

PS cores

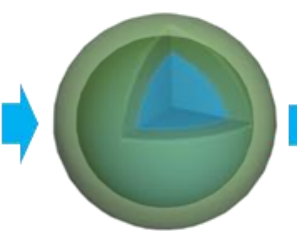

Hybrid $\mathrm{SiO}_{2}$ shell

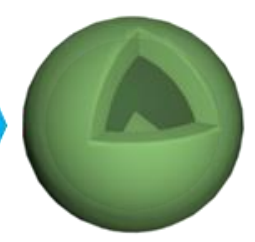

Hollow $\mathrm{SiO}_{2}$ particles
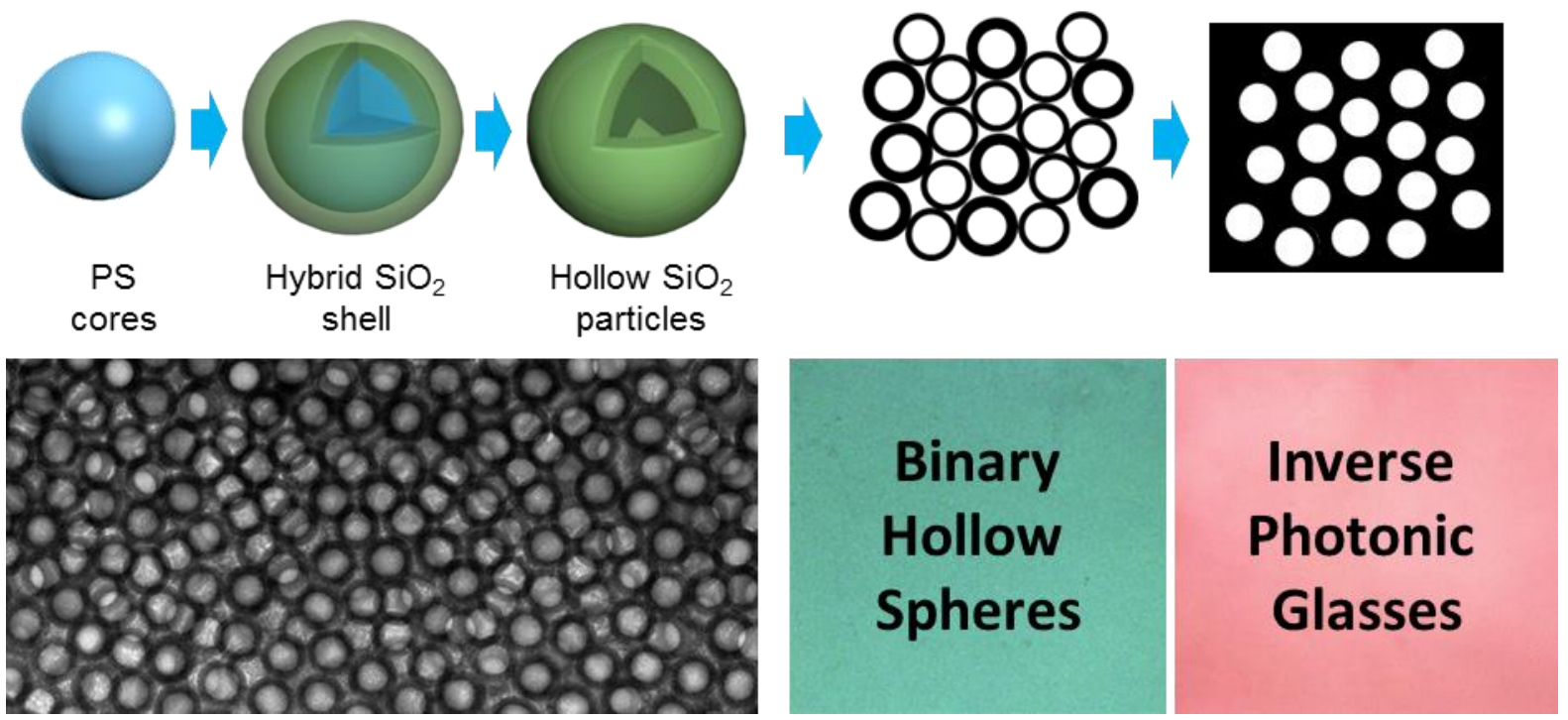


\section{Abstract}

A major fabrication challenge is producing disordered photonic materials with angleindependent structural red color. Theoretical work has shown that such color can be produced by fabricating inverse photonic glasses with monodisperse, non-touching voids in a silica matrix. Here we demonstrate a route toward such materials and show that they have angleindependent red color. We first synthesize monodisperse hollow silica particles with precisely controlled shell thickness, then make glassy colloidal structures by mixing two types of hollow particles with the same core size and different shell thicknesses. We then infiltrate the interstices with index-matched polymers, producing disordered porous materials with uniform, non-touching air voids. This procedure allows us to control the light-scattering form factor and structure factor of these porous materials independently, which is not possible to do in photonic glasses consisting of packed solid particles. The structure factor can be controlled by the shell thickness, which sets the distance between pores, while the pore size determines the peak wavevector of the form factor, which can be set below the visible range to keep the main structural color pure. By using a binary mixture of 246-nm and 268-nm hollow silica particles with 180 -nm cores in an index-matched polymer matrix, we achieve angle-independent red color that can be tuned by controlling the shell thickness. Importantly, the width of the reflection peak can be kept constant, even for larger inter-particle distances.

Keywords: Photonic glass, Hollow silica, Inverse, Templates, Self-assembly 


\section{Introduction}

Inspired by nanostructures in bird feathers or firefly lantern, ${ }^{1-3}$ a new kind of structurally colored material, called a "photonic glass," has recently been developed. ${ }^{4-6}$ Unlike photonic crystals, which show iridescent colors - that is, colors that depend on both the viewing angle and the orientation of the sample - photonic glasses can have angle-independent colors that are indistinguishable from traditional dyes or pigments.

One way to produce such materials is by quickly drying colloidal suspensions. ${ }^{7-9}$ However, this approach yields only blue or green colors, and never red. Magkiriadou et al. ${ }^{10}$ used a single-scattering model to explain why red is difficult to achieve: When colloidal glasses scatter light, two peaks arise in the reflection spectra: one comes from the form factor and is determined primarily by the size and refractive index of individual particles, while the other comes from the structure factor and is determined primarily by the inter-particle distance. ${ }^{11}$ However, in glassy packings of spheres these two peaks cannot be independently controlled, because the inter-particle spacing is determined by the particle size. When the sphere size is such that the peak of the structure factor corresponds to a reflected green or blue wavelength, the peak of the form factor is in the ultraviolet (UV) and cannot be seen by the human eye. If one increases the sphere size, so as to increase the average inter-particle distance and thus shift the peak of the structure factor wavelengths, the peak of the form factor enters the visible region, making the structural color a mix of blue and red that appears magenta. ${ }^{12-14}$

We seek to create a red, angle-independent, pure structural color from a colloidal sample. By "red" we mean that there is a single peak in the red part of the reflection spectrum (about 600-700 nm). Samples that show a secondary peak at short wavelengths are not red, but 
instead appear magenta, as noted above. Thus, we seek to eliminate the second peak entirely. By "angle-independent" we mean that the color does not shift with the viewing angle and orientation of the sample. Finally, by "pure structural color," we mean that the color arises from the process of interference of light scattered from the sample, and not from absorption. Many recent papers discuss the use of melanin or similar materials in concert with colloidal assembly to create red colors. ${ }^{15-19}$ The melanin absorbs blue light, ${ }^{15-16}$ which can eliminate the secondary peak in the blue. Here we seek to avoid the use of melanin and other dyes, because wavelength-specific absorption is the basis of traditional color. Structural color is special because it relies on wavelength-dependent interference. We believe the distinction is important because structural color has a number of advantages over traditional color in applications - it is in principle resistant to fading, and a number of different colors can be made using the same materials. These advantages are lessened with the use of wavelengthdependent absorption. Thus, we seek to produce a "pure" structural color using only nonabsorbing particles and wavelength-independent absorption. Moreover, we aim to make materials in which not only the position, but also the width of the structural color peak can be controlled, independently of one another. To our knowledge, this feature has not been demonstrated previously.

One way to make red, angle-independent, red structural color is to decouple the structure and form factors so that the peak of the structure factor is in the visible but the peak of the form factor remains in the UV region. Park et al. showed that this can be done by making a photonic glass consisting of PS-poly( $N$-isopropylacrylamide- $c o$-AAc) core-shell particles. ${ }^{20}$ The hydrogel shell can be swollen with so much water that it has approximately the same index of refraction as the fluid between the spheres. Thus, the form factor is controlled almost 
entirely by the cores, and the interparticle distance by the shells. By using small PS cores, the peak of the form factor can be placed in the UV, and by using large shells, the peak of the structure factor can be shifted to the red (or any other color). A similar approach was developed earlier by Ueno et al., who used small silica particles coated with PMMA stabilizer to form disordered colloidal structures in an ionic liquid, resulting in angleindependent structural colors of blue, green, and red. ${ }^{21}$ In both of these approaches, however, the particles must remain dispersed in the liquid. It is not possible to dry the composites to make solid pieces of photonic glasses, which may be needed for applications such as coatings or paints. Recently, Yang and her colleagues reported structurally colored materials formed by spheres with bumpy shells. However, this approach did not completely separate the form factor from the structure factor. ${ }^{22}$

A different approach to red, angle-independent, pure structural color that does not require a fluid medium relies on using low-refractive-index particles in a high-index matrix. Magkiriadou et al. ${ }^{23}$ showed that the low refractive index inside the particles reduces the optical path length of light traveling within them, thus allowing the form factor to be shifted to the UV. However, for optimal red color the low-refractive-index particles should not touch one another.

Packings of such particles are called "inverse photonic glasses," and several methods have been developed to make them. For example, small particles can be embedded in a medium and then removed, leaving disordered porous materials; examples include polystyrene (PS) particles embedded in a silica matrix and then dissolved ${ }^{10}$ and silica particles that are dispersed in a photo-curable resin that is then cross-linked, leaving a composite with a 
disordered packing of silica particles that can be etched away. ${ }^{24}$ Both approaches yield red colors as predicted. In these "kinetic" approaches where the colloidal glass is formed in a reactive medium that is solidified, the inter-particle distance is controlled by the volume fraction. To keep the particles from touching one another, the volume fractions must be low, and thus the peak of the structure factor is typically broader than that obtained in a dense random packing, resulting in less saturated colors at longer wavelengths.

In this report, we describe a method for making solid inverse photonic glasses where the width of the reflection peak does not change with wavelength. We achieve this by packing hollow spheres and then filling the interstices with a polymer that is index-matched to the sphere shells. The hollow particles are prepared by coating polystyrene beads with organically modified silane, then burning off the polystyrene. This procedure allows us to precisely control the shell thickness between $20 \mathrm{~nm}$ and $50 \mathrm{~nm}$. By changing the thickness of the shells, we can change the average spacing between the voids within them, and thus we control the color. Because the volume fraction of the spheres is the same for all colors, the width of the peak in the structure factor does not change. Furthermore, the small size of the empty core ensures that the peak of the form factor is in the UV. To avoid crystallization, we use a binary mixture of hollow spheres with nearly equal sizes and a stoichiometric ratio near 1:1. ${ }^{25}$ This fabrication method produces dry samples with uniform air voids that are not interconnected, which Magkiriadou et al. ${ }^{23}$ showed is important for shifting the peak of the form factor to the UV and keeping the second peak of the structure factor in the visible.

\section{Results and Discussion}

Synthesis of hollow silica particles. 
We prepare monodisperse hollow particles by coating silica on polystyrene (PS) particles and burning out the organic part. For consistent formation of disordered structures, it is important to make monodisperse hollow particles with controlled shell thickness. There are many experimental approaches to prepare uniform hollow silica particles, ${ }^{26-30}$ which mostly involve sol-gel reactions on latex particles. However, it is still challenging to prepare dispersions of monodisperse hollow spheres, because the particles may aggregate into dimers during shell growth or during the calcination process that removes the cores. Moreover, it is not a trivial task to re-disperse them after heat treatment, which can significantly sinter the particles together.
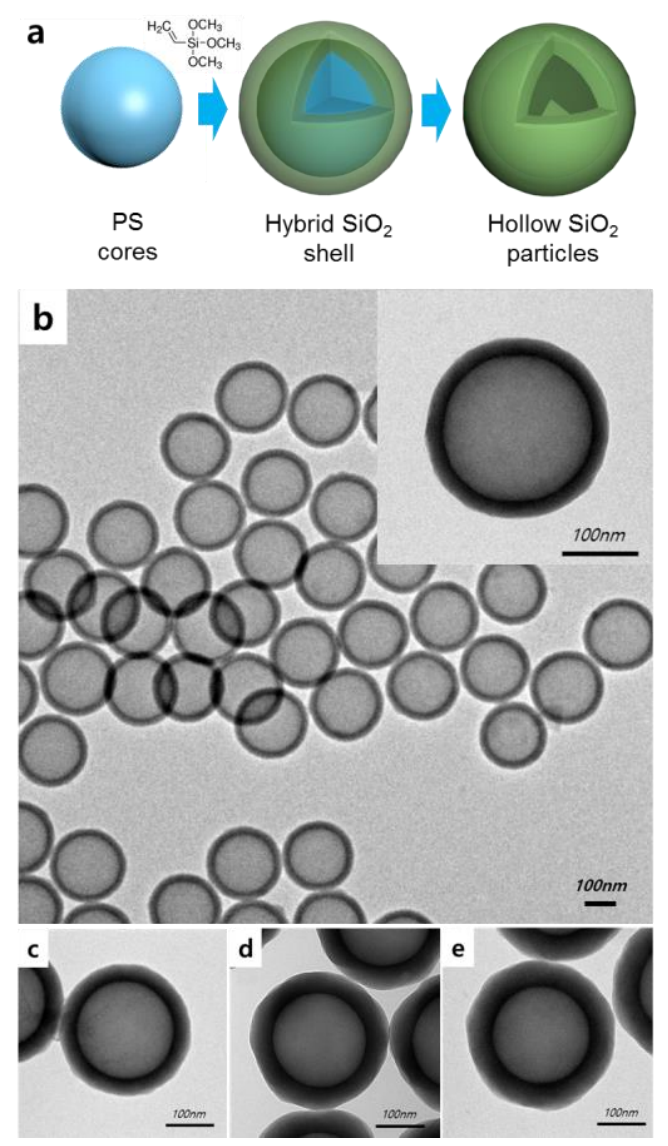

Figure 1. (a) Synthetic route to hollow silica spheres from polystyrene spheres in which vinyltrimethoxysilane (VTMS) is coated on the polystyrene beads by a sol-gel reaction. (b-e) 
TEM images of hollow silica particles with $180 \mathrm{~nm}$ cores and (b) $16 \mathrm{~nm}$ (c) $31 \mathrm{~nm}$ (d) $40 \mathrm{~nm}$ (e) $44 \mathrm{~nm}$ shell thickness.

To circumvent these problems, we used vinyltrimethoxysilane (VTMS) as a precursor for the silica shell, which is hydrolyzed and condensed onto the polymer particles. ${ }^{31}$ When a few VTMS precursors are condensed and form oligomers, they are much more hydrophobic than oligomers of alkoxide precursors such as tetraethylorthosilicate (TEOS) or tetramethylorthosilicate (TMOS), used in previously reported methods. ${ }^{26-30}$ At low concentration, the VTMS oligomers can be precipitated on the polymeric particles to form a uniform organosilica shell. Therefore, the shells form quickly and at relatively neutral $\mathrm{pH}$, which prevents aggregation of particles during the coating process. Once the organosilica shell is formed around the PS particles, we age and dry them in a freeze-drier to minimize the contact between particles. Subsequently, we burn out the PS part during heat treatment, yielding monodisperse silica particles (see schematic, Figure 1a).

As shown in Figure 1b, we prepared PS beads (200-nm) by surfactant-free emulsion polymerization and coated with VTMS. Then, we anneal freeze-dried powders of these coreshell particles at $600{ }^{\circ} \mathrm{C}$ for 6 hours. By adding more VTMS, we can control the shell thickness from $16 \mathrm{~nm}$ to $44 \mathrm{~nm}$, as shown in Figure 1b-e and Figure S1. As shown in Figure $\mathrm{S} 2$, the diameter and shell thickness of hollow $\mathrm{SiO}_{2}$ particles have a narrow distribution: the relative standard deviation (the ratio of the standard deviation to the mean, $\alpha=\sigma / \mu$ ) is less than 0.009 for all particle diameters.

\section{Disordered colloidal structures from binary hollow silica particles.}

Among hollow silica particles with various shell thicknesses, we choose a set of binary 1:1 
colloidal mixtures by number of particle that, as shown in previous work, ${ }^{9,25,32}$ yields a disordered structure and not a binary crystal. We also add carbon nanotube (CNT, $\sim 0.003 \mathrm{wt} \%$ in colloidal glass) to suppress multiple scattering over a broad range of wavelengths. ${ }^{7}$ Notably, the average size of the binary mixture corresponds to the core-to-core distance $(d)$, which determines the wavelength inside the material $(\lambda \approx 2 d)$ corresponding to the peak of the structure factor in backscattering $(\theta=\pi)$. The peak wavevector $(q)$ of the structure factor is determined from the following equation ${ }^{9-10}$ :

$$
q=\frac{4 \pi}{\lambda} \sin \frac{\theta(=\pi)}{2}=\frac{4 \pi}{\lambda} \approx \frac{4 \pi}{2 d}=\frac{2 \pi}{d}
$$

By evaporating the water from a binary colloidal mixture of 242-nm and 268-nm particles, we assemble a disordered structure with a peak wavevector $(q)$ around $24.4 \mu \mathrm{m}^{-1}$ (Figure $2 \mathrm{~b}$ ), which is close to the calculated value $\left(24.6 \mu \mathrm{m}^{-1}\right)$ from Equation (1) by taking $d$ as the weight- average diameter $\left(d_{\text {avg }}=d_{\mathrm{s}} \times \alpha_{s}+d_{l} \times\left(1-\alpha_{s}\right)\right.$, where $\alpha_{\mathrm{s}}$ is the number density of small hollow silica spheres to large ones). Similarly, a disordered structure of 212-nm and 242-nm hollow silica particles has a wavevector $(q)$ around $27.9 \mu \mathrm{m}^{-1}$ (Figure S3b), which is close to the calculated value $\left(27.7 \mu \mathrm{m}^{-1}\right)$.

We fill the interstices between the hollow spheres with index-matched photo-curable monomer (trimethylolpropane ethoxylate triacrylate, TMPEOTA, $n=1.46)^{33}$, which produces an optically homogeneous matrix surrounding the disordered pores, as shown in the crosssectional TEM image of Figure 2c. By inspecting broken samples of composites of hollow silica and TMPEOTA (see SEM image in Figure 2d), we infer that the internal structures are disordered and that the TMPEOTA infiltrates the colloidal glasses completely. 


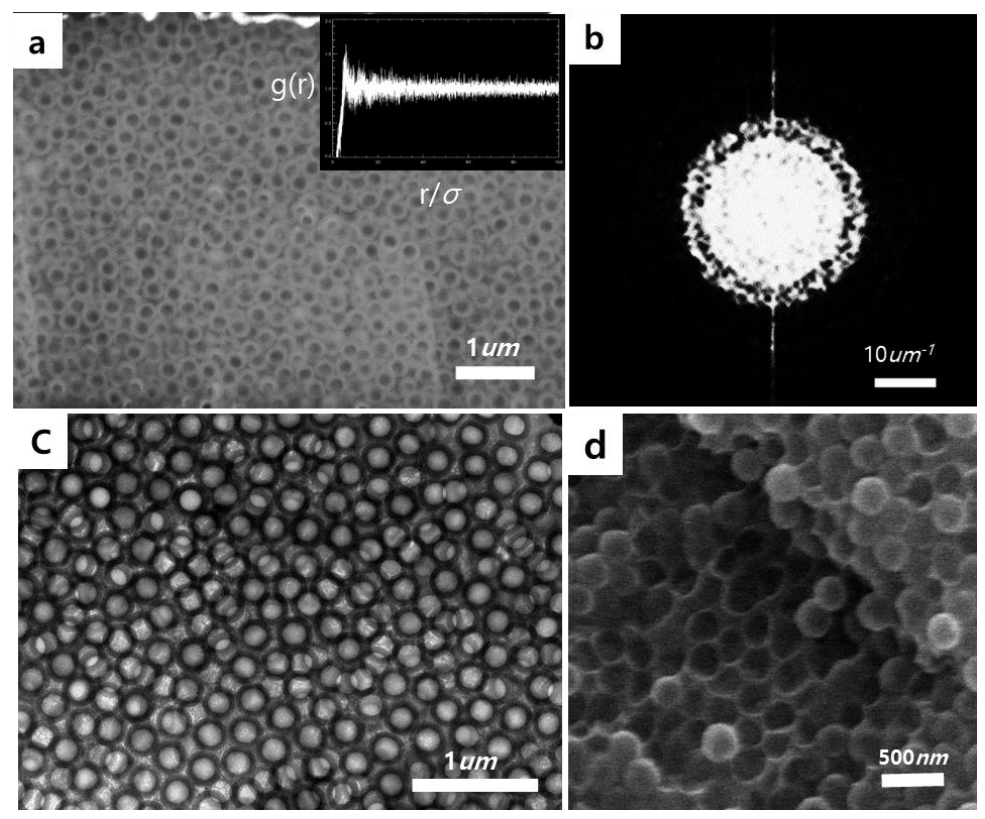

Figure 2. (a) SEM image of colloidal glasses of bi-disperse hollow silica particles with same cores $(180 \mathrm{~nm})$ but different shell sizes and (b) Fourier-transformed image. (c) TEM images of the top surface and (d) cross-sectional SEM image of composites of inverse colloidal glasses consisting of TMPEOTA infiltrating between the hollow spheres. Dimples are formed where hollow spheres have detached.

Photonic glasses formed from a binary colloidal mixture of 242-nm and 268-nm hollow silica particles with 180-nm cores show green color (Figure 3a), but after infiltration with indexmatching polymer, the color shifts to red (Figure 3b). The positions of the peaks agree with estimates from the single scattering model of Magkiriadou et al. The average inter-particle size in a binary mixture with 1:1 mixing ratio is $255 \mathrm{~nm}$, from which the peak of the structure factor can be estimated from $\lambda=2 n_{\mathrm{eff}} d$. The effective refractive index ( $\left.n_{\mathrm{eff}}\right)$ in a glassy packing of hollow spheres is 1.176 , as calculated from the Maxwell-Garnett theory ${ }^{34,35}$ assuming that the packing fraction $\phi_{p}$ is $0.637\left(\phi_{p}=\phi_{p}^{*}+c_{1} \delta+c_{2} S \delta^{2} \text {, Table S1 }\right)^{36}$ :

$$
n_{e f f}=n_{s} \sqrt{\frac{2 n_{s}^{2}+n_{\text {air }}^{2}+2 \emptyset_{p}\left(\frac{V_{\text {core }}}{V_{P-\text { avg }}}\right)\left(n_{\text {air }}^{2}-n_{s}^{2}\right)}{2 n_{s}^{2}+n_{\text {air }}^{2}-\emptyset_{p}\left(\frac{V_{\text {core }}}{V_{P-\text { avg }}}\right)\left(n_{\text {air }}^{2}-n_{s}^{2}\right)}}
$$


where $n_{s}$ is the refractive index of the material surrounding the particles, $n_{\text {air }}$ is the refractive index of the air, $V_{\mathrm{p} \text {-avg }}$ is the average volume of hollow particles, and $V_{\text {air }}$ is core volume of hollow particle. Thus, we find that the predicted peak is at $599 \mathrm{~nm}$ for this binary mixture. When it is infiltrated with polymer, the sample has an effective refractive index ( $\left.n_{\mathrm{eff}}\right)$ of 1.356 , yielding a peak at $691 \mathrm{~nm}$. These calculated values are close to our measurement, shown in Figure 3e.
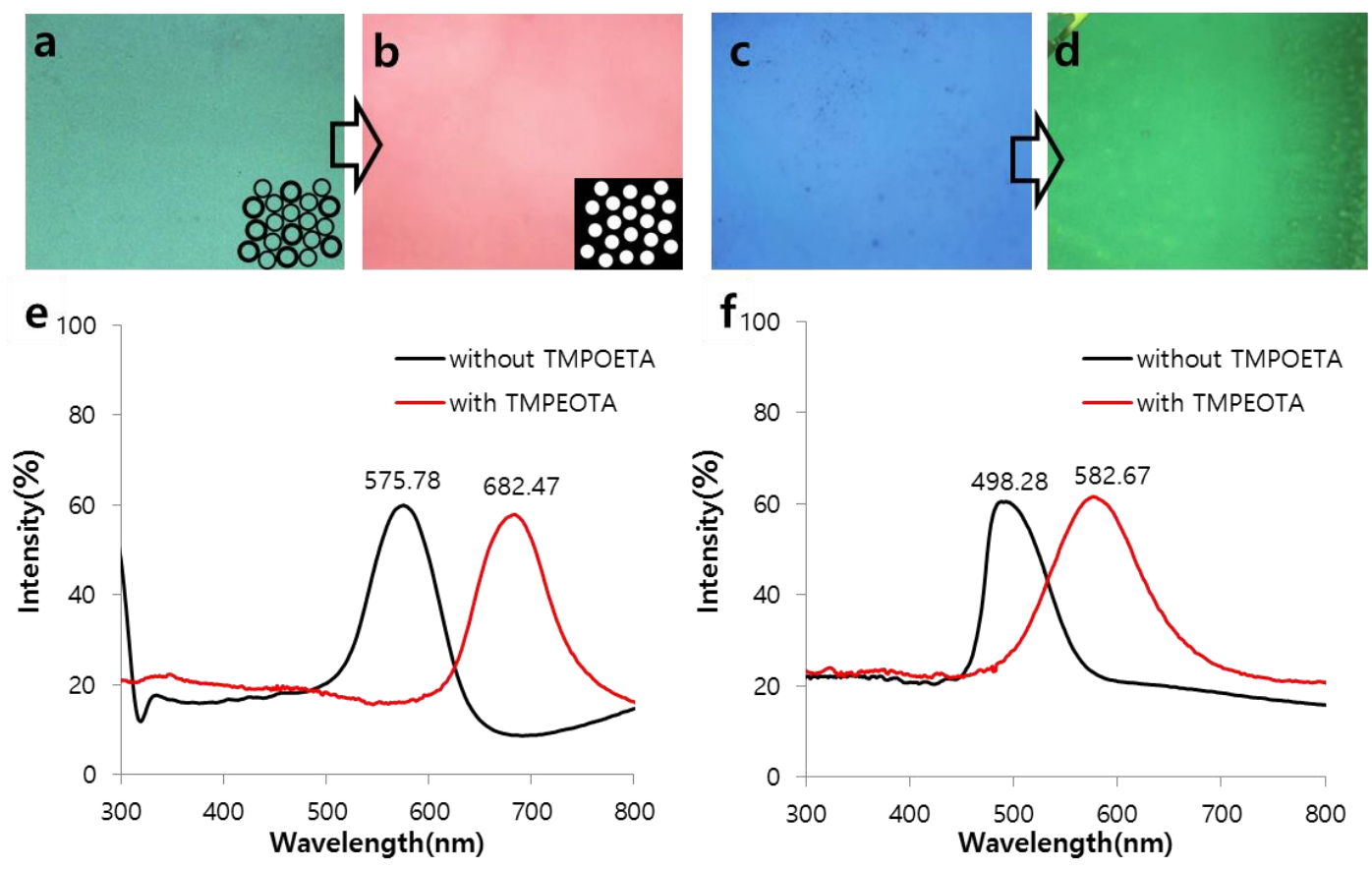

Figure 3. Colors of samples as seen in reflection-mode optical microscopy. Samples consist of glasses of bidisperse hollow silica particles containing (a) 242-nm and 268-nm-diameter spheres that in (b) are infiltrated with TMPEOTA; and (c) 212-nm and 242-nm-diameter spheres that in (d) are infiltrated with TMPEOTA. Reflection spectra of colloidal glasses (black line) and composites with TMPEOTA (red line) with (e) a mixture of 242-nm and 268$\mathrm{nm}$ - diameter particles and (f) a mixture of 212-nm and 242-nm-diameter particles.

Similarly, we find that photonic glasses formed from a binary mixture of 212-nm and 242-nm hollow silica particles with 180-nm cores show blue colors that turn into green colors when the glasses are filled with index-matching polymer (Figure 3d). The predicted peak of the reflection spectrum is $515 \mathrm{~nm}$ for the binary mixture and $596 \mathrm{~nm}$ for the infiltrated sample. 
These peak values are close to the $498 \mathrm{~nm}$ and $583 \mathrm{~nm}$ peaks that we observe in the reflection spectrum shown in Figure 3f.

We note also that, in the angle-independent red sample shown in Figure 3b, there is no secondary peak at short wavelengths, indicating that we have indeed successfully moved the form factor peak into the UV. Furthermore, the saturation of the color, as determined from the ratio of the peak reflectivity (at $682 \mathrm{~nm}$, red line) to the reflectivity at short wavelengths in Figure $3 \mathrm{e}$, is $2.79\left(I_{\max } / I_{\text {background }}=57.83 / 20.71\right)$. The saturation for colloidal glasses with a mixture of 212-nm and 242-nm-diameter particles (Figure 3f, red line, $582 \mathrm{~nm}$ ) is 2.61 (61.27/23.51). These saturation values are significantly higher than those previously reported in the literature for systems with angle-independent structural red colors. ${ }^{15,16,37}$

\section{Angle-independent Colors from Inverse Photonic Glasses.}

To test whether our samples do indeed show angle-independent structural color, we measure, at various angles, the reflection spectrum of a binary mixture of 242-nm and 268-nm hollow silica particles with 180 -nm cores, infiltrated with index-matched resin. At $0^{\circ}$ and $45^{\circ}$ viewing angles, we observe red colors, as shown in Figure 4a and 4b. The reflection spectra, shown in Figure $4 \mathrm{c}$, confirm that the peaks remain around $680 \mathrm{~nm}$ for viewing angles from $0^{\circ}$ to $60^{\circ}$. In case of crystalline porous materials out of 268-nm hollow silica particles with $180-\mathrm{nm}$ cores, reflectivity in $0^{\circ}$ viewing angle is relatively high but at other viewing angle, we can clearly see angle-dependency of reflection color and intensity as shown in Figure S4.

In the "kinetic" approach to make glassy structures that we described earlier, relatively low concentrations of particles are needed to obtain isotropic reflective red color, which can lead 
to broadening of the distribution of inter-particle distances and of the width of the reflection peak. By contrast, in our system of binary core-shell particles, the inter-particle distance has a narrow distribution regardless of the pore volume fraction, because the volume fraction of the core-shell particles is always the same. Therefore, the peak width in the reflection spectra is consistent as we shift the peak wavelength, as shown in Figures $3 \mathrm{e}, 3 \mathrm{f}, 4 \mathrm{c}$. The consistency of the width of the peak is important for controlling the color in applications, and is the key to obtaining the high values of the saturation that we have demonstrated.

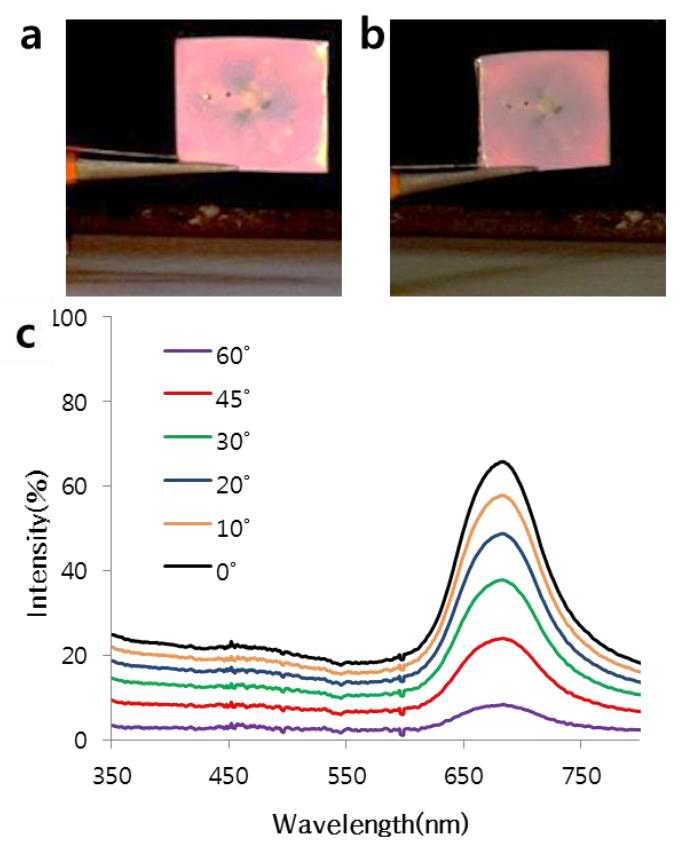

Figure 4. Photographs of inverse colloidal glass films at (a) $0^{\circ}$ and (b) $45^{\circ}$ viewing angles, and (c) corresponding reflection spectra at various viewing angles ranging from $0^{\circ}$ to $60^{\circ}$.

\section{Conclusion}

We have synthesized monodisperse hollow silica particles in which the shell thickness is precisely controlled. By mixing two types of hollow particles with the same core size but different shell sizes, condensing them into glassy aggregates, and infiltrating the interstices 
with index-matched polymers, we produce disordered porous materials with uniform, nontouching air voids. Unlike in photonic glasses made by packing solid particles, in our structures the form factor and structure factor can be independently controlled, since the pore size can be tuned independently of the shell thickness. By making small pores, we can shift the peak from the form factor below the visible range, and by changing the average shell thickness we can control the peak from the structure factor. In addition to controlling the location of the peak of the reflection spectrum, we can also control its width, set by the width of the distribution of the shell thicknesses. We have shown that this method leads to angleindependent, pure structural colors, including red colors. These structural colors can potentially be used as materials in solar cells, ${ }^{38}$ reflective displays, or as fade-resistant color particles for signage, cosmetics, or random lasers. ${ }^{39-40}$ 


\section{Materials and Methods}

\section{Materials}

Styrene (>97\%, Sigma-Aldrich), potassium persulfate (KPS, Sigma-Aldrich), sodium styrene sulfonate (NaSS, JUNSEI), sodium hydrogen carbonate, $\mathrm{NaHCO}_{3}$, JUNSEI), inhibitor removers (Sigma Aldrich), vinyltrimethoxysilane (VTMS, 98\%, Sigma-Aldrich), ammonium hydroxide solution $\left(\mathrm{NH}_{4} \mathrm{OH}, 28-30 \%\right.$, Sigma-Aldrich), trimethylolpropane ethoxy triacrylate (TMPEOTA, SK CYTEC), Darocur 1173 (SK CYTEC).

\section{Synthesis of PS particles}

Monodisperse polystyrene (PS) particles are synthesized by surfactant-free emulsion polymerization. Typically, at first, the polymerization inhibitor (hydroquinone) or stabilizer in the monomer are removed using an inhibitor remover packed column (Sigma-Aldrich). Then, $50 \mathrm{~g}$ of styrene monomer are mixed at $900 \mathrm{rpm}$ with $300 \mathrm{~g}$ of deionized water under nitrogen gas. After $30 \mathrm{~min}$ stirring, $50 \mathrm{~g}$ of co-monomer solution $(0.5 \mathrm{wt} \%$ of NaSS in water) and $50 \mathrm{~g}$ of buffer solution ( $1 \mathrm{wt} \%$ of $\mathrm{NaHCO}_{3}$ in water) are added to the mixture of styrene and water. Finally, $50 \mathrm{~g}$ of free-radical initiator solution ( $1 \mathrm{wt} \%$ of KPS in water) are added after another $30 \mathrm{~min}$ stirring. The reaction proceeds at $80{ }^{\circ} \mathrm{C}$ for $12 \mathrm{~h}$ producing monodisperse PS particles in water. After the complete reaction, un-reacted chemicals and secondary nuclei (or small particles) are removed by several centrifugation/re-dispersion cycles.

\section{Preparation of hollow silica particles}

The PS colloidal particles are diluted to $1 \mathrm{wt} \%$ in DI water. Ammonium hydroxide solution $(28-30 \mathrm{wt} \%, 0.7 \mathrm{ml})$ is added to the PS particle suspension $(10 \mathrm{ml})$ and mixed at $600 \mathrm{rpm}$ for 
30 min. Organosilane with vinyl groups (VTMS, $0.4-2.0 \mathrm{ml}$ ) is mixed with DI water and hydrolyzed by stirring at $900 \mathrm{rpm}$ for $30 \mathrm{~min}$. The PS particle suspension and hydrolyzed VTMS solution are mixed and stirred at $600 \mathrm{rpm}$ for $6 \mathrm{~h}$ at room temperature. After the solgel coating of organosilica on PS particles, unreacted precursors are removed by a vacuumfiltration process using a membrane filter with 200-nm pores (Millipore, JUNG-IL Science Co., Ltd.). After washing by centrifugation and re-dispersion several times, the core-shell particles are freeze-dried on a substrate and annealed at $600{ }^{\circ} \mathrm{C}$ for $6 \mathrm{~h}$ to remove the polymer from the cores and organic chemicals from the shells and to densify the silica shell.

\section{Fabrication of inverse photonic glasses}

Inverse photonic glass structures are prepared by packing two different sizes of hollow silica particles and infiltrating the interstices of the glass with polymer resin (TMPEOTA). Typically, two hollow silica particle suspensions (30 wt\%) $246 \mathrm{~nm}$ and $268 \mathrm{~nm}$ in diameter are mixed by dispersing powders in water. Next, a CNT solution $(0.1 \mathrm{wt} \%, 10 \mu \mathrm{l})$ is added to the hollow silica suspensions in water $(1 \mathrm{ml}) .{ }^{41}$ The mixture is dried at $40{ }^{\circ} \mathrm{C}$ for $12 \mathrm{~h}$. After the drying, TMPOETA $(100 \mu \mathrm{l})$ with photoinitiator $(0.02 \mathrm{wt} \%$, Darocur 1173$)$ is added to the dried colloidal glasses and then solidified by UV curing.

\section{Characterization}

Colloidal particles are observed under scanning electron microscopy (SEM, Hitachi, S-4300) and transmission electron microscopy (TEM, JEOL LTD, JEM-2100F) to measure the size and determine structure. Cross-sectional TEM samples of inverse photonic glass are prepared using a focused ion beam machine (FIB, LYRA3 XMU, TESCAN). The color of photonic colloidal glasses is observed under a reflection-mode optical microscope (Nikon, Ecipse 80i), 
and their reflectivity is measured using a fiber-coupled spectrometer (Ocean Optics Inc., BH2000-BAL).

\section{Supporting Information}

Additional data for SEM images of the hollow $\mathrm{SiO}_{2}$ particles with air core (Figure $\mathrm{S} 1$ ), Histogram of size and shell thickness of hollow $\mathrm{SiO}_{2}$ particles (Figure S2), TEM image and FFT image of colloidal glasses (Figure S3), Reflection spectra of crystalline porous materials (Figure S4), Table of the theoretical equations for calculating the packing density value (Table S1).

\section{Acknowledgements}

We acknowledge support from the National Research Foundation of Korea (NRF) (NRF2010-0029409 and NRF-2014M3A9B8023471), the Small and Medium Business Administration of Korea (SMBA) under grant number 10050334 (World-Class 300 Project), the Harvard MRSEC, funded by the National Science Foundation under grant number DMR1420570, and the Xerox University Affairs Committee. 


\section{References}

(1) Chen, L.; Shi, X.; Li, M.; Hu, J.; Sun, S.; Su, B.; Wen, Y.; Han, D.; Jiang, L.; Song, Y. Bioinspired Photonic Structure by the Reflector Layer of Firefly Lantern for Highly Efficient Chemiluminescence Sci. Rep. 2015, 5, 12965.

(2) Noh, H.; Liew, S. -F.; Saranathan, V.; Mochrie, S. G. J.; Prum, R. O.; Dufresne, E. R.; Cao, H. How Noniridescent Colors are Generated by Quasi-Ordered Structures of Bird Feathers Adv. Mater. 2010, 22 2871-2880.

(3) Saranathan, V., Forster, J. D.; Noh, H.; Liew, S.-F.; Mochrie, S. G. J.; Cao, H.;

Dufresne, E. R.; Prum, R. O. Structure and Optical Function of Amorphous Photonic Nanostructures from Avian Feather Barbs: A Comparative Small Angle X-Ray Scattering (SAXS) Analysis of 230 Bird Species J. R. Soc., Interface. 2012, 9, 25632580.

(4) Shi, L.; Zhang, Y.; Dong, B.; Zhan, T.; Liu, X.; Zi, J. Amorphous Photonic Crystals with Only Short-Range Order Adv. Mater. 2013, 25, 5314-5320.

(5) Ballato, J. Tailoring Visible Photonic Bandgaps Through Microstructural Order and Coupled Material Effects in $\mathrm{SiO}_{2}$ Colloidal Crystals J. Opt. Soc. Am. B 2000, 17, 219225.

(6) Takeoka, Y. Angle-Independent Structural Coloured Amorphous Arrays J. Mater. Chem. 2012, 22, 23299-23309.

(7) García, P. D.; Sapienza, R.; Blanco, Á.; López, C. Photonic Glass: A Novel Random Material for Light Adv. Mater. 2007, 19, 2597-2602.

(8) Espinha, A.; Ibisate, M.; Blanco, Á.; López, C. Engineering the Light-Transport Mean Free Path in Silica Photonic Glasses Part. Part. Syst. Charact. 2016, 33, 352-357. 
(9) Forster, J. D.; Noh, H.; Liew, S. F.; Saranathan, V.; Schreck, C. F.; Yang, L.; Park, J. G.; Prum, R. O.; Mochrie, S. G.; O'Hern, C. S. Biomimetic Isotropic Nanostructures for Structural Coloration Adv. Mater. 2010, 22, 2939-2944.

(10) Magkiriadou, S.; Park, J.-G.; Kim, Y.-S.; Manoharan, V. N. Disordered Packings of Core-Shell Particles with Angle-Independent Structural Colors Opt. Mater. Express. 2012, 2, 1343-1352.

(11) Bohren, C. F.; Huffman, D. R. Absorption and Scattering of Light by Small Particles; John Wiley \& Sons: New York, 1983

(12) Wang, F.; Zhang, X., Lin, Y.; Wang, L.; Zhu, J. Structural Coloration Pigments based on Carbon Modified ZnS@SiO2 Nanospheres with Low-Angle Dependence, High Color Saturation, and Enhanced Stability ACS Appl. Mater. Interfaces 2016, 8, 5009-5016.

(13) Iwata, M.; Teshima, M.; Seki, T.; Yoshioka, S.; Takeoka, Y. Bio-Inspired Bright Structurally Colored Colloidal Amorphous Array Enhanced by Controlling Thickness and Black Background Adv. Mater. 2017, DOI: 10.1002/adma.201605050.

(14) Katagiri, K.; Tanaka, Y.; Uemura, K.; Inumaru, K.; Seki, T.; Takeoka Y. Structural color coating films composed of an amorphous array of colloidal particles via electrophoretic deposition NPG Asia Materials 2017, 9, e355.

(15) Xiao, M.; Li, Y.; Allen, M. C.; Deheyn, D. D.; Yue, X.; Zhao, J. Gianneschi, N. C.; Shawkey, M. D.; Dhinojwala, A. Bio-Inspired Structural Colors Produced via SelfAssembly of Synthetic Melanin Nanoparticles ACS Nano, 2015, 9, 5454-5460.

(16) Zhang, Y.; Dong, B,; Chen, A.; Liu, X.; Shi, L.; Zi, J. Using Cuttlefish Ink as an Additive to Produce Non-iridescent Structural Colors of High Color Visibility Adv. Mater. 2015, $27,4719-4724$.

(17) Kawamura, A.; Kohri, M.; Yoshioka, S.; Taniguchi, T.; Kishikawa, K. Structural Color 
Tuning: Mixing Melanin-Like Particles with Different Diameters to Create Neutral Colors Langmuir 2017, 33, 3824-3830.

(18) Kawamura, A.; Kohri, M.; Morimoto, G.; Nannichi, Y.; Taniguchi, T.; Kishikawa, K. Full-Color Biomimetic Photonic Materials with Iridescent and Non-Iridescent Structural Colors Sci. Rep. 2016, 6, 33984.

(19) Kohri, M.; Nannichi, Y.; Taniguchi, T.; Kishikawa, K. Biomimetic non-iridescent structural color materials from polydopamine black particles that mimic melanin granules J. Mater. Chem. C 2015, 3, 720-724.

(20) Park, J. G.; Kim, S. H.; Magkiriadou, S.; Choi, T. M.; Kim, Y. S.; Manoharan, V. N. FullSpectrum Photonic Pigments with Non-iridescent Structural Colors through Colloidal Assembly Angew. Chem., Int. Ed. 2014, 53, 2899-2903.

(21) Ueno, K.; Sano, Y.; Inaba, A.; Kondoh, M.; Watanabe, M. Soft Glassy Colloidal Arrays in an Ionic Liquid: Colloidal Glass Transition, Ionic Transport, and Structural Color in Relation to Microstructure J. Phys. Chem. B. 2010, 114, 13095-13103.

(22) Yang, X.; Ge, D.; Wu, G.; Liao, Z.; Yang, S. ACS Appl. Mater. Interfaces. 2016, 8, 16289-16295.

(23) Magkiriadou, S.; Park, J.-G.; Kim, Y.-S.; Manoharan, V. N. Absence of Red Structural Color in Photonic Glasses, Bird Feathers, and Certain Beetles Phys. Rev. E 2014, 90, 062302.

(24) Lee, G. H.; Sim, J. Y.; Kim, S.-H. Polymeric Inverse Glasses for Development of Noniridescent Structural Colors in Full Visible Range ACS Appl. Mater. Interfaces. 2016, $8,12473-12480$.

(25) Palberg, T.; Bartsch, E.; Beyer, R.; Hofmann, M.; Lorenz, N.; Marquis, J.; Niu, R.; Okubo, T. To Make a Glass_-Avoid the Crystal J. Stat. Mech.: Theory Exp. 2016, 
074007.

(26) Nandiyanto, A. B. D.; Akane, Y.; Ogi, T.; Okuyama, K. Mesopore-Free Hollow Silica Particles with Controllable Diameter and Shell Thickness via Additive-Free Synthesis Langmuir 2012, 28, 8616-8624.

(27) Ernawati, L.; Ogi, T.; Balgis, R.; Okuyama, K.; Stucki, M.; Hess, S. C.; Stark, W. J. Hollow Silica as an Optically Transparent and Thermally Insulating Polymer Additive Langmuir 2016, 32, 338-345.

(28) Chen, W.; Takai, C.; Khosroshahi, H. R.; Fuji, M.; Shirai, T. Surfactant-Free Fabrication of $\mathrm{SiO}_{2}$-Coated Negatively Charged Polymer Beads and Monodisperse Hollow $\mathrm{SiO}_{2}$ Particles Colloids Surf., A. 2015, 481, 375-383.

(29) Zhang, L.; D’Acunzi, M.; Kappl, M.; Auernhammer, G. n. K.; Vollmer, D.; van Kats, C. M.; van Blaaderen, A. Hollow Silica Spheres: Synthesis and Mechanical Properties Langmuir 2009, 25, 2711-2717.

(30) Gao, T.; Jelle, B. P.; Sandberg, L. I. C.; Gustavsen, A. Monodisperse Hollow Silica Nanospheres for Nano Insulation Materials: Synthesis, Characterization, and Life Cycle Assessment ACS Appl. Mater. Interfaces. 2013, 5, 761-767.

(31) Rhee, D. K.; Jung, B.; Kim, Y. H.; Yeo, S. J.; Choi, S.-J.; Rauf, A.; Han, S.; Yi, G.-R.; Lee, D.; Yoo, P. J. Particle-Nested Inverse Opal Structures as Hierarchically Structured Large-Scale Membranes with Tunable Separation Properties ACS Appl. Mater. Interfaces. 2014, 6, 9950-9954.

(32) Hunt, N.; Jardine, R.; Bartlett, P. Superlattice Formation in Mixtures of Hard-Sphere Colloids Phys. Rev. E 2000, 62, 900-913.

(33) Widmann, J. f.; Davis, E. J. Photochemical Initiated Polymerization of Single Microdroplets Colloid. Polym. Sci. 1996, 274, 525-531. 
(34) J. C. Maxwell Garnett, Colours in Metal Glasses and in Metallic Films Philos. Trans. R. Soc. London, A 1904, 203, 385-420.

(35) Hutchinson, N. j .; Coquil, T.; Navid, A.; Pilon, L. Effective Optical Properties of Highly Ordered Mesoporous Thin Films Thin Solid Films 2010, 518, 2141-2146.

(36) Desmond, K. W.; Weeks, E. R. Influence of Particle Size Distribution on Random Close Packing of Spheres Phys. Rev. E 2014, 90, 022204.

(37) Ge, D.; Yang, L.; Wu, G.; Yang, S. Angle-Independent Colours from Spray Coated Quasi-Amorphous Arrays of Nanoparticles: Combination of Constructive Interference and Rayleigh Scattering J. Mater. Chem. C. 2014, 2, 4395-4400.

(38) Vynck, K.; Burresi, M.; Riboli, F.; Wiersma, D. S. Photon Management In TwoDimensional Disordered Media Nat. Mater. 2012, 11, 1017-1022.

(39) Wiersma, D. S. Optical Physics: Clear Directions for Random Lasers Nature 2016, 539, 360-361.

(40) Wiersma, D. S. The Physics and Applications of Random Lasers Nat. Phys. 2008, 4, 359-367.

(41) Wang, Y.; Iqbal, Z.; Mitra, S. Rapidly Functionalized, Water-Dispersed Carbon Nanotubes at High Concentration J. Am. Chem. Soc. 2006, 128, 95-99. 
Supporting Information

\section{Inverse Photonic Glasses by Packing Bi-disperse}

\section{Hollow Microspheres with Uniform Cores}

Seung-Hyun Kim, ${ }^{\dagger}$ Sofia Magkiriadou, ${ }^{\S}$ Do Kyung Rhee, ${ }^{\dagger}$ Doo-Sung Lee ${ }^{\dagger}$, Pil J. Yoo,,$\dagger$ Vinothan N. Manoharan, ${ }^{\#, ~ G i-R a ~ Y i ~}{ }^{\dagger} *$

${ }^{\dagger}$ School of Chemical Engineering and ${ }^{\dagger}$ Sungkyun Advanced Institute of Nano Technology (SAINT), Sungkyunkwan University, Suwon 16419 Republic of Korea

${ }^{\S}$ Department of Physics and ${ }^{\#} J o h n$ A. Paulson School of Engineering and Applied Sciences, Harvard University, Cambridge, USA

*E-mail: yigira@skku.edu 

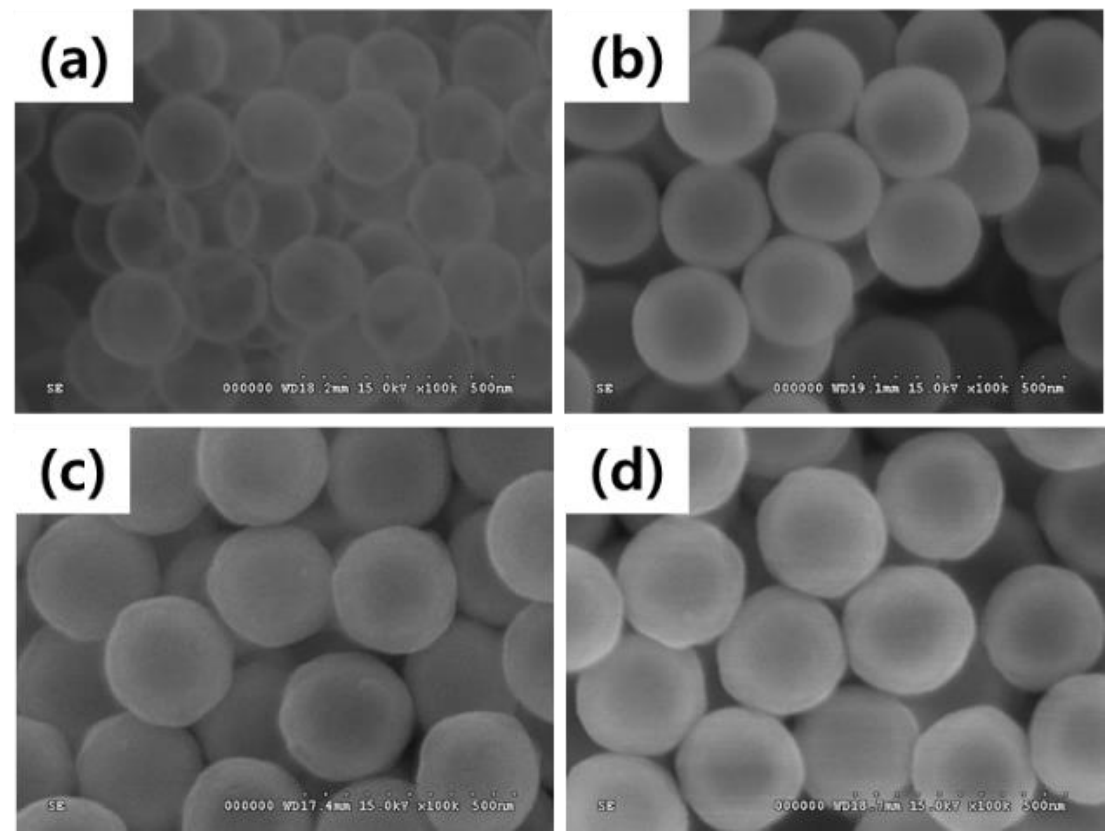

Figure S1. SEM images of hollow $\mathrm{SiO}_{2}$ particles with $180-\mathrm{nm}$ air core and (a) $16 \mathrm{~nm}$ (b) 31 $\mathrm{nm}$ (c) $40 \mathrm{~nm}$ (d) $44 \mathrm{~nm} \mathrm{SiO}_{2}$ shells. 

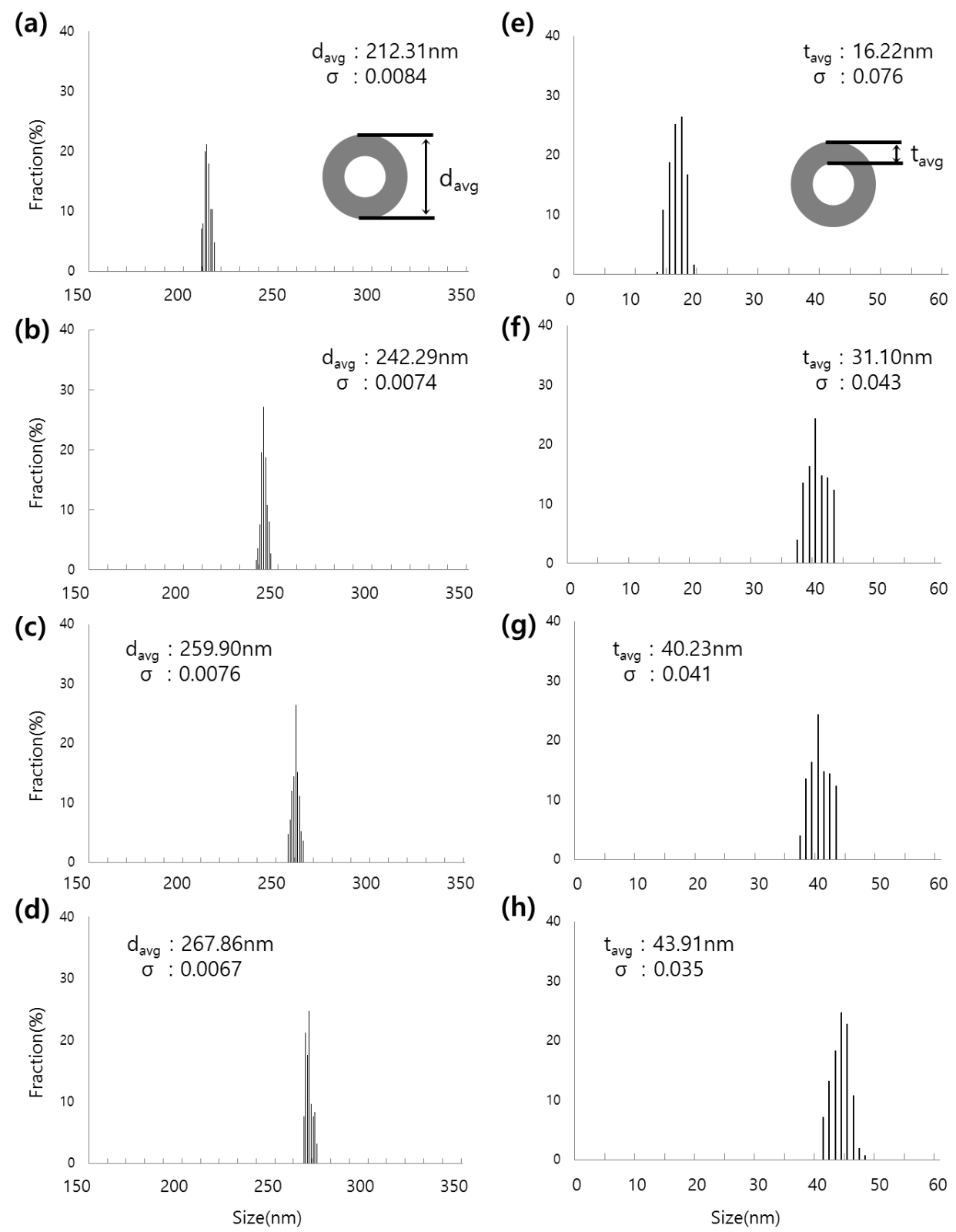

Figure S2. Histogram of size and shell thickness of hollow silica particles with average diameter ( $d_{a v g}$ ) of (a) $212.31 \mathrm{~nm}$, (b) $242.29 \mathrm{~nm}$, (c) $259.90 \mathrm{~nm}$, and (d) $267.86 \mathrm{~nm}$. Average shell thicknesses ( $\mathrm{t}_{\text {avg }}$ ) are (e) $16.22 \mathrm{~nm}$ (f) $31.10 \mathrm{~nm}(\mathrm{~g}) 40.23 \mathrm{~nm}$ and $(\mathrm{h}) 43.91 \mathrm{~nm}$. Average diameter $\left(\mathrm{d}_{\mathrm{avg}}\right)$, shell thickness $\left(\mathrm{t}_{\mathrm{avg}}\right)$ and the relative standard deviation $(\sigma)$ are determined by measuring 250 particles in TEM images. 

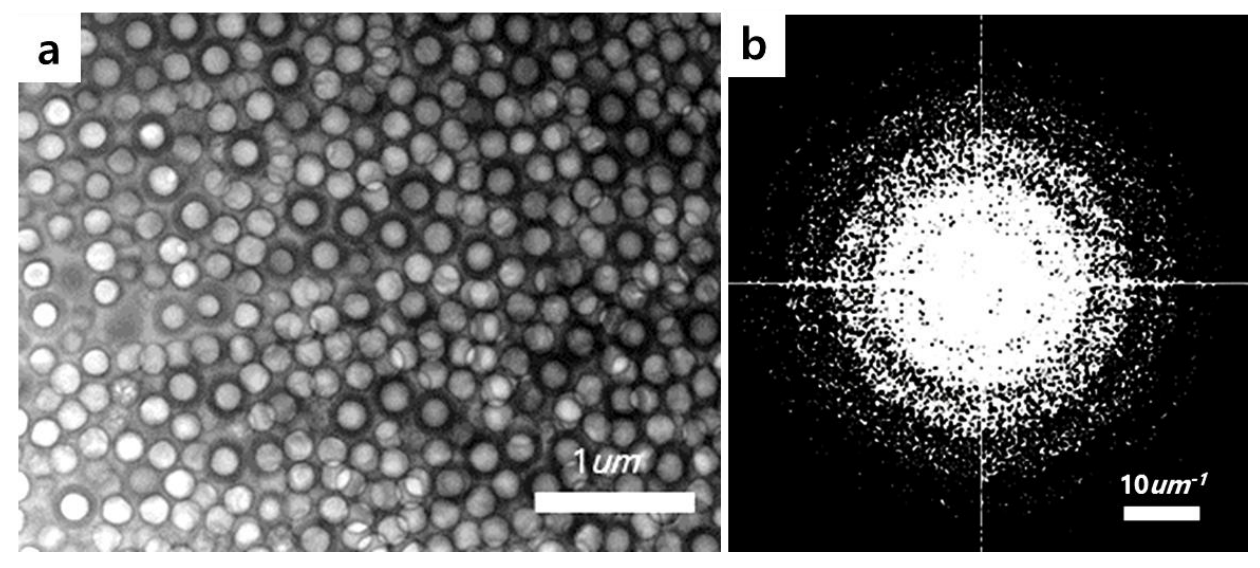

Figure S3. TEM image of (a) colloidal glasses of bi-disperse 212-nm and 242-nm hollow silica particles with same cores $(180 \mathrm{~nm})$ but different shell sizes and (b) Fourier-transformed image. 


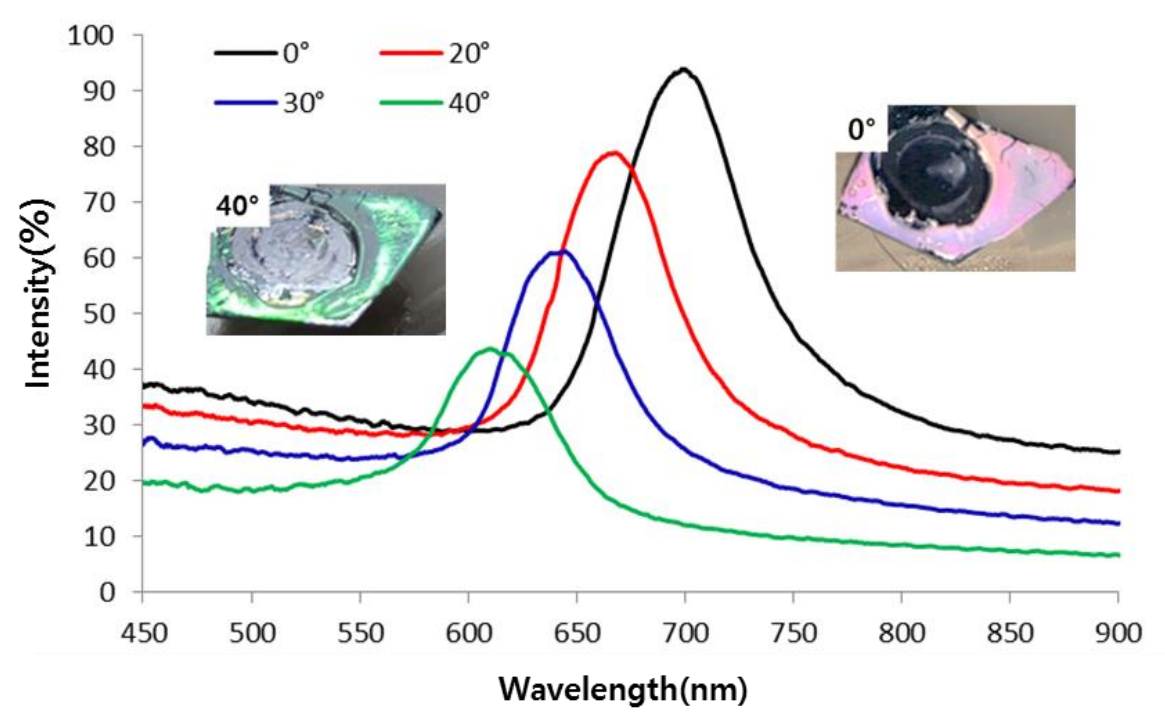

Figure S4. Reflection spectra of crystalline porous materials by assembling 268-nm hollow silica particles with 180 -nm cores in index-matched polymer matrix at various viewing angles from $0^{\circ}$ to $40^{\circ}$. Inset images show reflection color at $0^{\circ}$ and $40^{\circ}$ viewing angles. 
Table S1. Summary of the theoretical equation for calculating the packing density value. Here $\alpha_{l}$ is the number density of large hollow silica spheres and $\alpha_{s}$ is the number density of small hollow silica spheres.

\begin{tabular}{|c|c|}
\hline Packing density & $\phi_{p}=\phi_{p}^{*}+c_{1} \delta+c_{2} S \delta^{2}$ (where $\left.\phi_{p}^{*}=0.634, c_{1}=0.0658, c_{2}=0.0857\right)$ \\
\hline Polydispersity & $\delta=\left[(1-\rho)(\mathrm{a}-1)^{2}+\rho(\mathrm{b}-1)^{2}\right]^{1 / 2}$ \\
\hline Skewness & $\left.\mathrm{S}=\left[(1-\rho)(\mathrm{a}-1)^{3}+\rho(\mathrm{b}-1)^{3}\right] / \delta^{3}\right)$ \\
\hline Constrained & $\mathrm{a}=1 /(1-\rho+\eta \rho), \quad \mathrm{b}=\eta /(1-\rho+\eta \rho)$ \\
\hline Number fraction & $\rho=\alpha_{l} / \alpha_{s}+\alpha_{l}$ \\
\hline Size ratio & $\eta=\mathrm{d}_{l} / \mathrm{d}_{s}$ \\
\hline
\end{tabular}

